\title{
Impairment of context memory by $\beta$-amyloid peptide in terrestrial snail
}

\author{
Tatiana A. Korshunova, Natalia I. Bravarenko and Pavel M. Balaban* \\ Institute of Higher Nervous Activity and Neurophysiology, Russian Academy of Sciences, Moscow, Russia \\ Edited by: $\quad$ Martin Giurfa, Research Centre on Animal Cognition, CNRS - University Paul Sabatier, Toulouse, France \\ Reviewed by: Makoto Mizunami, Graduate School of Life Science, Tohoku University, Sendai, Japan \\ Martin Giurfa, Research Centre on Animal Cognition, CNRS - University Paul Sabatier, Toulouse, France
}

\begin{abstract}
We examined influence of the $\beta$-amyloid peptide ( $\beta A P)$ (25-35) neurotoxic fragment on Helix lucorum food-aversion learning. Testing with aversively conditioned carrot showed that 2,5 and 14 days after training the $\beta A P$-injected group responded in a significantly larger number of cases and with a significantly smaller latency than the sham-injected control group. The results demonstrate that the AP partially impairs the learning process. In an attempt to specify what component of memory is impaired we compared responses in a context in which the snails were aversively trained, and in a neutral context. It was found that the sham-injected learned snails significantly less frequently took the aversively conditioned food in the context in which the snails were shocked, while the $\beta A P-$ injected snails remembered the aversive context 2 days after associative training, but were not able to distinguish two contexts 5 , and 14 days after training. In a separate series of experiments a specific context was associated with electric shock, and changes in general responsiveness were tested in two contexts several days later. It was found that the $\beta A P$-injected snails significantly increased withdrawal responses in all tested contexts, while the sham-injected control animals selectively increased responsiveness only in the context in which they were reinforced with electric shocks. These results demonstrate that the $\beta A P(25-35)$ interferes with the learning process, and may play a significant role in behavioral plasticity and memory by selectively impairing only one component of memory the context memory.
\end{abstract}

Keywords: Alzheimer's disease, animal model, behavior, synaptic plasticity, snail

\section{INTRODUCTION}

Alzheimer's disease $(\mathrm{AD})$ is a neurodegenerative disorder affecting cognitive functions and memory. It is widely accepted that the accumulation of $\beta$-amyloid peptide ( $\beta$ AP), a small peptide which derives from the proteolytic processing of the amyloid precursor protein (APP), is central to the pathogenesis of disease (Bartoo et al., 1997; Naslund et al., 2000; Selkoe, 2000). Although the potential neurotoxic properties of $\beta$ AP have been known for over a decade, it is still not known which components of behavior change under $\beta \mathrm{AP}$, resulting in progressive cognitive decline.

It is clear from the available literature that the APP fragments, including $\beta A P$, can exert a powerful regulation of key neural functions including cell excitability, synaptic transmission and long-term potentiation, both acutely and on the longrun. Recently it was shown that neuronal activity modulates the formation and secretion of $\beta$ AP (Kamenetz et al., 2003) which is detected in nano-molar concentrations in healthy individuals throughout life both in the cerebrospinal fluid and

*Correspondence: Pavel M. Balaban, Institute of Higher Nervous Activity and Neurophysiology, Russian Academy of Sciences, Butlerova 5a, 117485 Moscow, Russia. e-mail: balaban@ihna.msk.ru

Received: 19 May 2008; paper pending published: 30 June 2008; accepted: 21 August 2008; published online: 02 September 2008.

Citation: Front. Behav. Neurosci. (2008) 2: 3. doi: 10.3389/neuro.08/003.2008

Copyright (c) 2008 Korshunova, Bravarenko and Balaban. This is an open-access article subject to an exclusive license agreement between the authors and the Frontiers Research Foundation, which permits unrestricted use, distribution, and reproduction in any medium, provided the original authors and source are credited. plasma (Seubert et al., 1992). It is thought that the $\beta$ AP plays a certain unknown role in normal physiology, including synaptic plasticity, learning and memory (Schulz, 1996; Stepanichev et al., 2004; Turner et al., 2003; Wu et al., 1995) Interestingly, APP and its homologues display a high degree of evolutionary conservation, and have been detected in all mammals, and also in some invertebrates (Johnstone et al., 1991; Wasco et al., 1995). Nevertheless, no Alzheimer-like pathology has ever been described in animals. In our previous experiments in terrestrial snails we described a possibility of changes elicited by application of $\beta \mathrm{AP}$ in neural responses and behavioral performance, and estimated effective concentrations of $\beta \mathrm{AP}$ (Samarova et al., 2005; Korshunova et al., 2007).

In the present paper, we examined in details influence of the neurotoxic AP fragment on food-aversion learning in terrestrial snail Helix. In behavioral experiments we compared responses of $\beta A P-i n j e c t e d$ and sham-injected snails to aversively conditioned food odor, and estimated context memory by testing responsiveness in reinforced and non-reinforced contexts.

Although studying the effects of BAP in snails does not make of this invertebrate a model of $\mathrm{AD}$, it may provide useful insights into the action mechanisms of certain components of Alzheimer pathology.

\section{MATERIALS AND METHODS SUBJECTS}

In the present series of experiments, 52 adult snails Helix lucorum L. (Crimea population) weighing 25-35 g were 
used. The snails were kept in active state at least 2 weeks before the experiment in a wet environment, and fed usually with carrots and cabbage. One to two weeks before the training session, the experimental animals were deprived of food. Between experimental sessions the subjects were housed in glass terraria.

Each animal was used only in one series of experiments. Experimental procedures are in compliance with the guide for the care and use of laboratory animals published by the National Institutes of Health (USA).

\section{TRAINING AND APPARATUS}

In the experimental set-up, the snail was tethered by its shell in a manner allowing it to crawl on a plastic ball that rotated freely in a water containing $0.01 \% \mathrm{NaCl}$ (Balaban, 1993). The ball was laced with bare stainless steel wire to complete an electrical circuit between the animal's foot and a carbon electrode placed in the water. This specific context was used in all behavioral experiments (context "ball"). As a different context we used in pilot experiments the flat glass lid of the terrarium that appeared to be a well distinguished context. In experiments taken for statistical evaluation we used a piece of glass $40 \mathrm{~cm} \times 60 \mathrm{~cm}$ as a different context ("glass"). Timing of accommodation in each context ( $5 \mathrm{~min}$ ), duration of testing, tactile stimulation, washing of ball and glass between tests were similar for both contexts. Before training each snail was code numbered, and the person testing them after training had no access to their history.

Electric shock was delivered (only in context "ball") using a 1-4 mA, $16 \mathrm{~Hz}$ square pulses of current through a macroelectrode applied manually to the dorsal surface of the snail's foot until a complete withdrawal of the snail to the shell. The reference carbon electrode was placed in the water in which the ball floated.

Punctate mechanical stimuli were applied with calibrated von Frey hairs permitting delivery of pressures ranging from 6 (estimated as weak) to 68 (noxious) $\mathrm{g} / \mathrm{mm}^{2}$. After several pilot series, the behavioral response, the intensity (tip diameter $0.2 \mathrm{~mm}, 25 \mathrm{~g} / \mathrm{mm}^{2}$ ) and the location of tactile stimulation were chosen. Ommatophore (posterior tentacles) withdrawal to tactile stimulation of the rostral part of the foot skin 4-5 mm behind the posterior tentacles appeared to be at the level of $20-40 \%$ of maximal in normal animals. In pilot experiments it was shown that responses to such test stimulation were sensitized after noxious stimuli, and this part of the foot skin was chosen as standard for tactile stimulation.

The snail's behavior was recorded with digital video camera. We analyzed video using software "PhysVis 1.4" (Kenyon College) available in the Internet. We scored the amplitude of tentacle withdrawal by measuring the distance between tip and base of the tentacle, and the latency of the food grasping. If in $120 \mathrm{~s}$ no food bite occurred, we stopped the test and scored the latency as $120 \mathrm{~s}$.

Before training, for 2 days each snail was exposed for $30 \mathrm{~min}$ daily to the experimental set-up. Twenty-four hours before the first training session snails from experimental group were injected with $\beta \mathrm{AP}\left(10^{-4} \mathrm{M}\right.$, total volume: $\left.0.2 \mathrm{ml}\right)$. Control animals received an injection of the same volume of saline. Three days later the same groups received additional injection of $\beta \mathrm{AP} /$ saline. Double blind testing was performed in all experiments.

\section{PROTOCOL 1: CONTEXT CONDITIONING}

After obtaining the pre-training scores in two contexts to test tactile stimuli (T0), snails of experimental and control groups (associative context conditioning) received two electrical shocks per day with at least $60 \mathrm{~min}$ intervals for 5 days in one environment only (ball) without any food. They were then tested (T1) using tactile stimulation 2 days after the training session in both environments (Figure 1A).

\section{PROTOCOL 2: FOOD-AVERSION CONDITIONING}

After obtaining the pre-training scores to test tactile stimuli in the two contexts, the first test session with food presentation was performed for two groups (session T0, Figure 2). During training, the snails of both experimental and control groups (associative food-aversion training) received two presentations of carrot per day associated with electric shocks with at least 60 min intervals for successive 14 days, and one presentation per day of cabbage (Figure 2). This type of training has been repeatedly shown to be associative in terrestrial snails (Balaban, 1993, 2002). Current was individually chosen for each snail so that a complete withdrawal of anterior part of the body was observed in response to a shock. No testing with tactile stimulation was performed during training days. Two, five and fourteen days after the training session (on the 3rd, 6th and 15th days, T1, T2 and $\mathrm{T} 3$, respectively), the responsiveness to food and to the same test tactile stimuli was separately scored (Figure 2). Double blind testing was performed in the two contexts. To reduce possible effects of recent handling, the test was administered no sooner than 5 min after the subjects had been placed in the environment. Only animals exhibiting active locomotion were tested. No shocks were delivered during the test sessions. If the snail did not bite the food positioned at a 5-mm distance from the lips during $120 \mathrm{~s}$ of presentation, a negative score was given.

\section{DRUG INJECTION}

The $\beta A P$ was dissolved (stock solution) in distilled water and stored at room temperature for at least $2 \mathrm{~h}$. The experimental snails were injected with $10^{-4} \mathrm{M}$ of $\beta \mathrm{AP}$ (injected volume $0.2 \mathrm{ml}$ : $0.1 \mathrm{ml}$ of amyloid stock solution $+0.1 \mathrm{ml}$ of saline). The control group received sham injection of $0.2 \mathrm{ml}$ of saline + distilled water. Injection was performed with a fine needle in non-sensitive part of the foot under the mantle bolster. During injection, the snail stopped locomotion, mostly because the shell was fixed by the experimenter, but showed no generalized withdrawal. We have not used a control peptide of similar size but different structure for two reasons: (1) any peptide may have its own specific effect while the aim of this study was to check whether or not the application of $\beta \mathrm{AP}$ induces changes in behavioral characteristics; (2) it is well known that isolated CNS releases many peptides in comparable concentrations (Kerkut, 1989).

\section{STATISTICAL ANALYSIS}

Mann-Whitney rank sum test was used to compare performance of two groups of snails. Wilcoxon signed-rank test was used for comparison of performance of the same group in different contexts. ANOVA on ranks was used for comparing several groups of data.

\section{RESULTS \\ CONTEXT CONDITIONING}

We examined the influence of $\beta$ AP on Helix context conditioning using the procedure described earlier (Balaban and Bravarenko, 1993). In all experiments, the snails from the experimental group were injected with $\beta \mathrm{AP}$ twice: $24 \mathrm{~h}$ before the first session and 3 days later. The control group received two sham injections of 
A

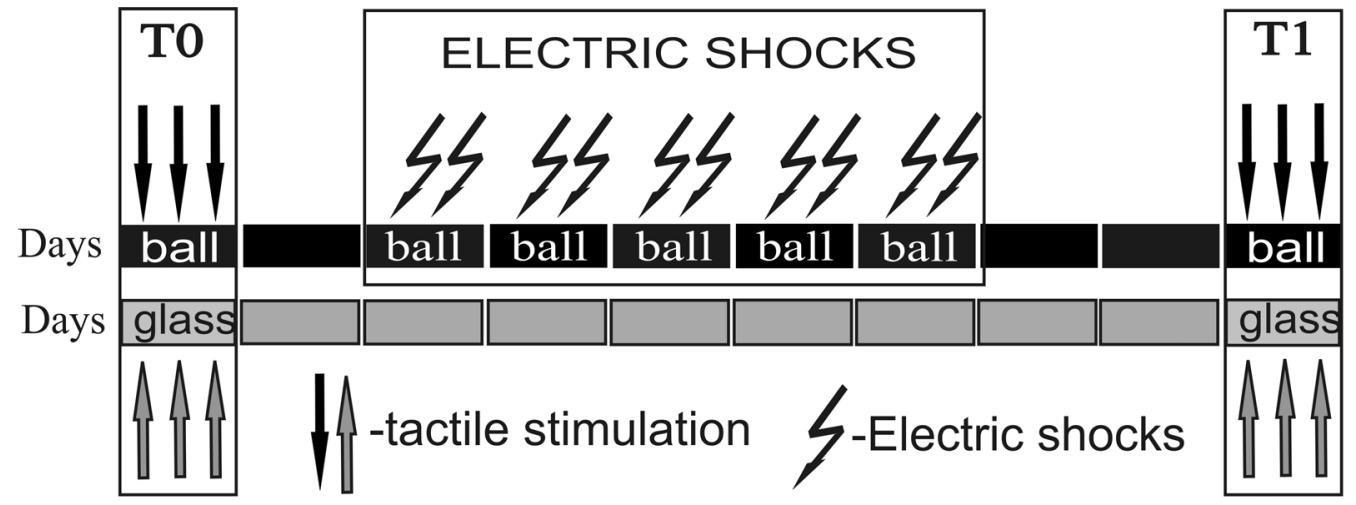

B

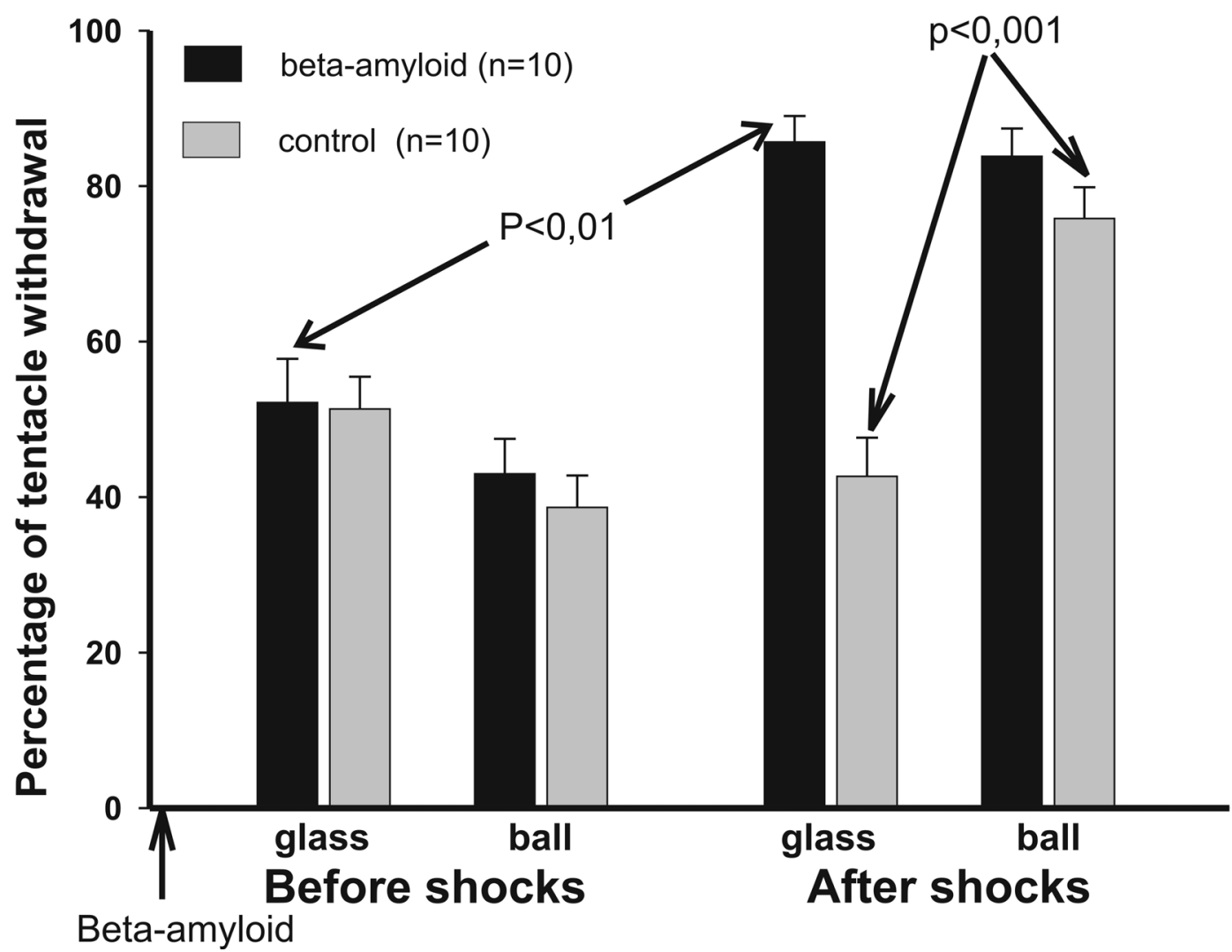

Figure 1 | Context conditioning. (A) Protocol of context conditioning experiments; electric shocks were given to both groups only in one context, on a ball. (B) Averaged amplitude of tentacle withdrawal to tactile stimuli in control snails $(n=10)$ and $\beta A P$-injected snails $(n=10)$ in two contexts: on the ball (reinforced context) and on the glass. Testing was performed before electric shocks (T0), and 2 days after the electric shocks (T1). $Y$-axis - amplitude of tentacle withdrawal in percentage of the length before the test.

saline at the same time. Snails from each group were shocked twice per day during 5 days, in the context "ball" only (see protocol on Figure 1A). Changes in responsiveness to a tactile stimulation of moderate intensity were scored in parallel in the sham-injected group of snails, and in snails injected with $\beta A P 1$ day before the 5-day sensitization session (T0) with electric shocks. Before sensitization, no significant differences were observed within or between groups (Figure 1B). Testing 2 days after the end of training (T1) demonstrated a significant increase $(p<0.01$, Wilcoxon signed-rank test) of amplitude of a withdrawal reaction to tactile test stimulation both in snails injected with $\beta \mathrm{AP}(n=10)$ and in the control group $(n=10)$. Scores taken after shocks evidenced that the $\beta A P$-injected snails shocked in one context significantly increased responsiveness in all tested contexts 2 days later, while the sham-injected animals selectively and significantly $(p<0.001$, Wilcoxon signed-rank test) increased responsiveness only in the context in which they were reinforced with electric shocks (Figure 1B). This result shows the presence of long-term associative (specific for the reinforced context) changes in withdrawal responses in the sham-injected snails, and a lack of associative connection in the $\beta A P$-injected animals with the context in which the snails were shocked.

\section{FOOD-AVERSION CONDITIONING}

All animals in these experiments (Figure 2), either sham $(n=15)$ or $\beta \mathrm{AP}(n=17)$, were injected twice: the day before the onset of 


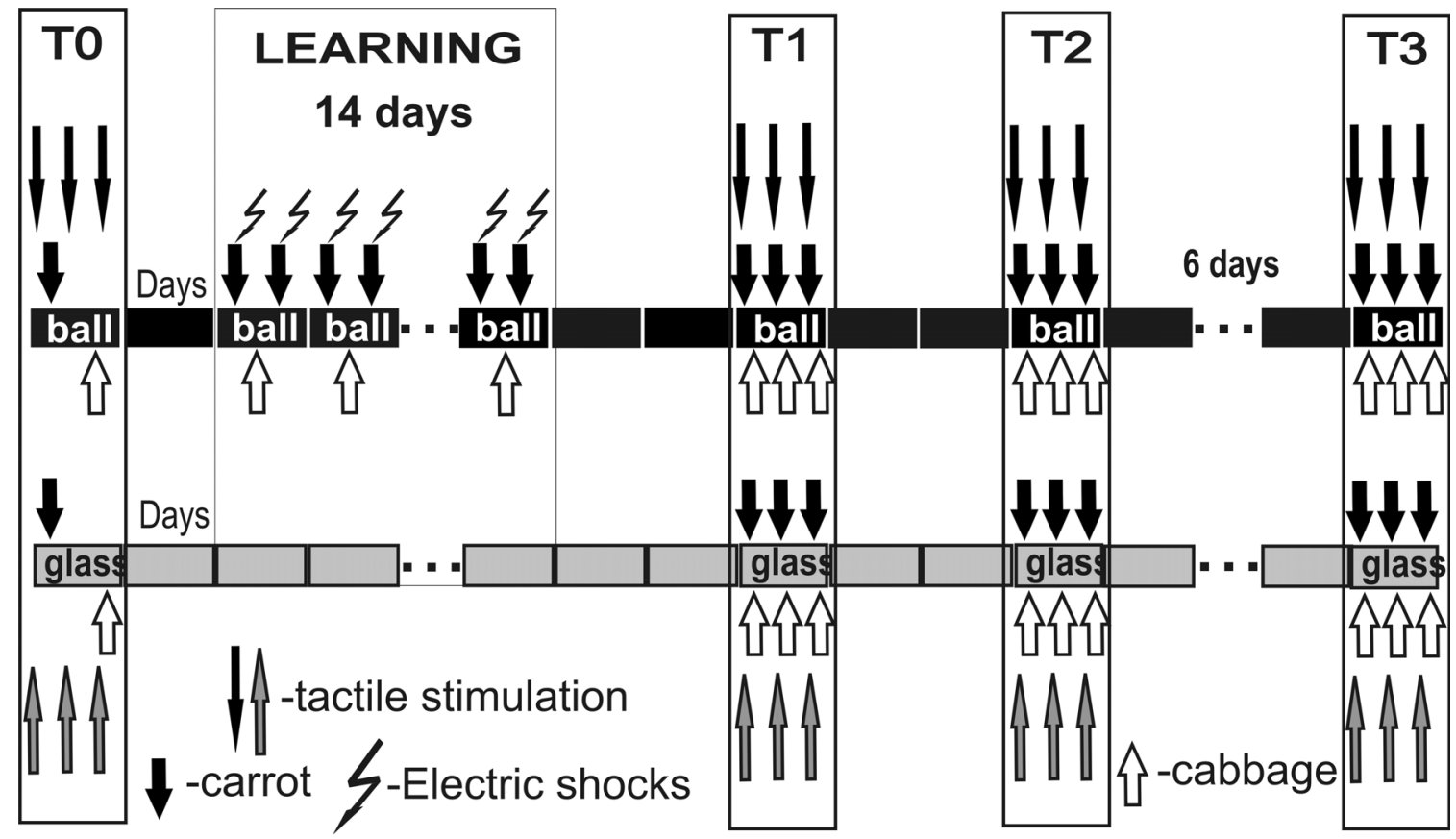

Figure 2 | Protocol of food-aversion conditioning experiments. Association of food (carrot) with electric shock was performed only in one context, while scoring of latency of food grasping to food presentation (carrot or cabbage) was performed in two contexts. Carrot presentation was used as a conditioned stimulus, while cabbage presentation never was associated with reinforcement. Testing of tentacle withdrawal to tactile stimulation, not associated with any reinforcement or context, in two contexts (glass and ball) allowed to estimate context memory independently of cued (food odor) memory.

test T0 and 3 days later. Dynamics of learning was estimated by scoring latency of food grasping during $120 \mathrm{~s}$ of food presentation in snails from experimental and control groups. Each grasping of one type of food (carrot, preferred food) was associated with electric shock, while the second type of food (cabbage) was never associated with shocks. Averaged latencies (Figure 3A) of food grasping demonstrated a steady increase during the training period (14 days). During the first 5 days of training, the latency scores were significantly $(p<0.05$, pairwise comparison, M.W.) higher in sham-injected snails than in $\beta$ AP-injected, but later, the latency scores were not significantly different $(p<0.2$, pairwise comparison, M.W., Figure $3 \mathrm{~A}$ ). Testing after 2, 5, and 14 days after the end of the training session revealed a significant difference $(p<0.01$, M.W. ) between the $\beta$ AP-injected and sham-injected animals in latency scores of reinforced food grasping, a fact reflecting significantly faster extinction of learning in experimental snails. Neither in the sham-injected group $(p<0.4$, ANOVA on ranks) nor in the $\beta$ AP-injected group $(p<0.1$, ANOVA on ranks, Figure 3B) did the latency of cabbage grasping (non-reinforced type of food) change significantly during training. Differences in responses to cabbage and carrot serve as an additional criterion for associativity of observed changes in behavior.

Scoring the latency of food grasping of associatively trained snails in two different contexts demonstrated better performance of sham-injected snails. The latency of carrot-grasping was significantly $(p<0.05$, M.W. $)$ smaller in $\beta$ AP-injected animals than in control ones in the reinforced context on the 3rd day after the end of training (after 2 days interval), while in the non-reinforced context (glass), the latency was similar in both groups (Figure 4A). Both groups demonstrated much higher latency scores after training $(p<0.001$, Wilcoxon signedrank test) in comparison with pre-training scores, a result reflecting generalized increase in responsiveness due to noxious reinforcement. Differences in response latencies in the two contexts remained highly significant $(p<0.001$, Wilcoxon signedrank test) in sham-injected animals, while in $\beta$ AP-injected snails this difference became smaller and non-significant $(p<0.7$, Wilcoxon signed-rank test) 14 days after the end of training (Figure 4A). The latency of cabbage grasping did not change significantly ( $p<0.4$, ANOVA on ranks) in both groups throughout all the experiment (Figures 3B and 4B), a fact that confirms the associative nature of the behavioral changes observed upon carrot presentation. These results suggest that the $\beta$ AP-injected snails learn and remember that they were shocked in the presence of a given food in a specific environment, but that this memory declines faster than in control animals.

An additional measurement describing learning is the percentage of carrot-grasping events during the $120 \mathrm{~s}$ criterion time. Before training, snails from both groups took food in $100 \%$ of the cases, while in the $3 r d$ day after training (T1) the sham-injected animals took food only in $6.6 \pm 3.5 \%$ of the cases in the reinforced context, and in $70.3 \pm 12.9 \%$ in the non-reinforced context $(p<0.001$, Wilcoxon signed-rank test). $\beta$ APinjected snails, on the other hand, took food in $37.2 \pm 8.9 \%$ in the reinforced context, and in $76.6 \pm 8.6 \%$ in the non-reinforced context $(p<0.01$, Wilcoxon, Figure $5 \mathrm{~A})$. The percentage of carrot-grasping events diminished in the 6th (T2) and 15th (T3) days after the end of training but was still significantly $(p<0.05$, Wilcoxon) different in the two contexts in the sham-injected snails, while it was not different any more in the $\beta$ AP-injected animals ( $p=0.84$ at T2, $p=0.126$ at T3, Wilcoxon, Figure 5A). These results highlight different characteristics of learning in $\beta A P-i n j e c t e d$ and sham-injected snails.

Finally, we tested changes in responsiveness to tactile stimulation of moderate intensity (not associated with any reinforcement) 

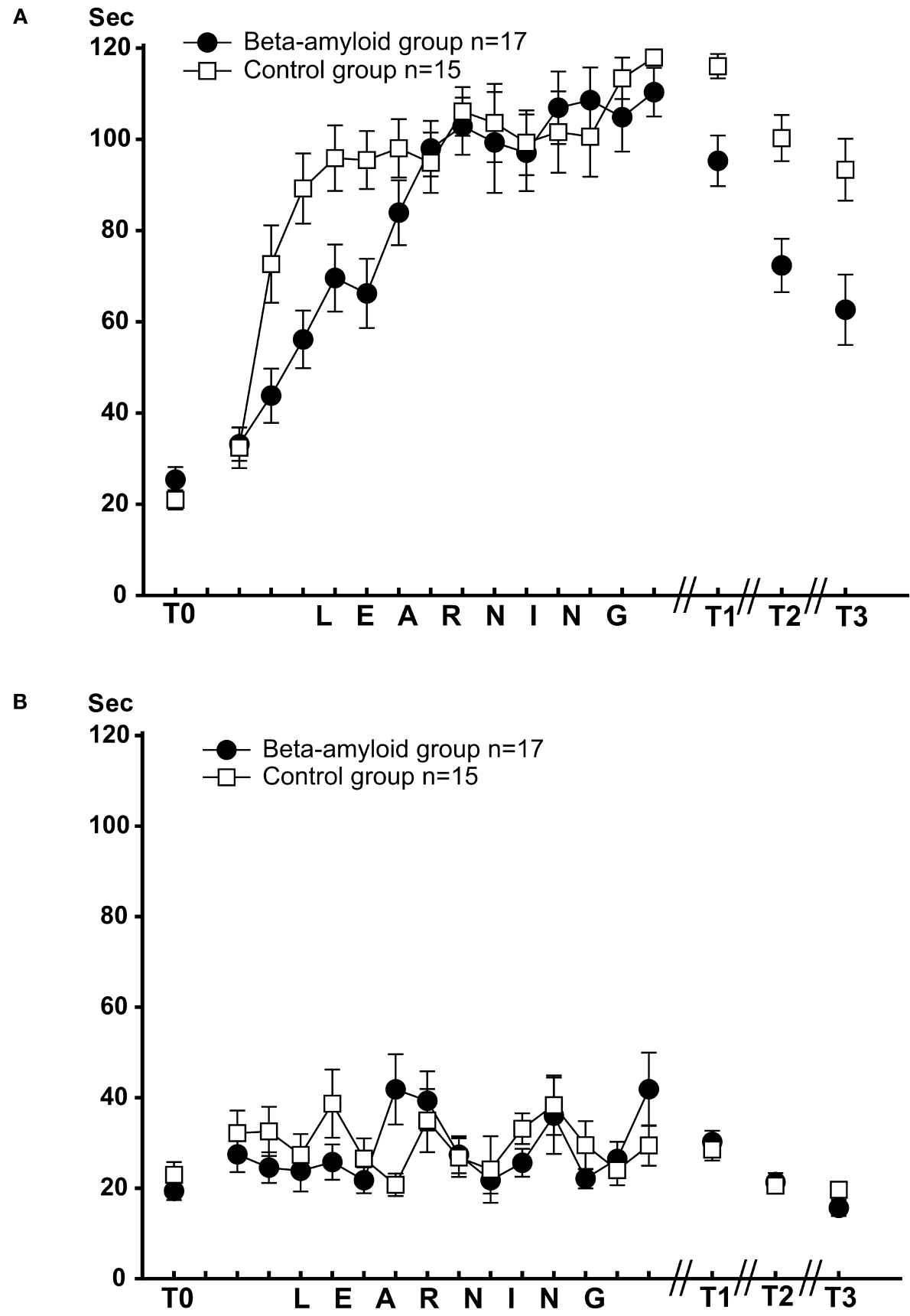

Figure 3 | Dynamics of food-aversion learning on the ball estimated by average latency of food grasping by the snail. ( $A$ ) Dynamics of carrot aversion learning in control snails $(n=15)$ and $\beta A P$-injected snails $(n=17) . X$-axis - days of experiment (see Figure 2 for detailed protocol), $Y$-axis - latency period

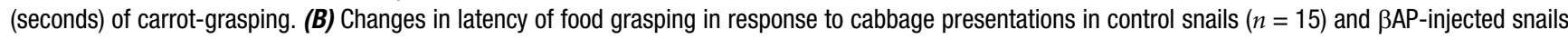
$(n=17) . X$-axis - days, $Y$-axis - latency period (seconds) of cabbage grasping.

in the two contexts in the same two groups of associatively trained animals. No difference between groups $(p<0.5$, M.W. $)$ was observed in the day prior to the training session (T0, Figure $5 \mathrm{~B})$. Testing in the 3rd day after the end of training (after 2 days of rest, T1) demonstrated a significant $(p<0.001$, Wilcoxon signedrank test) increase of amplitude in the withdrawal reaction to tactile test stimulation both in snails injected with $\beta$ AP $(n=10)$ and in the control group $(n=9)$ in the reinforced context. Still, in the non-reinforced context (glass), in the 3rd day after training, the responses of sham-injected snails were significantly weaker $(p<0.01$, Wilcoxon) than in the reinforced context, while no difference between contexts was noted in $\beta$ AP-injected animals $(p<0.4$, Wilcoxon, Figure 5B). This difference in behavioral performance between the $\beta \mathrm{AP}$-injected and sham-injected snails was maintained in the 6th and 15th days after the end of training, thus suggesting that the $\beta A P$-injected animals do not associate electric shocks with a particular context and exhibit a general increase in responsiveness. These results suggest that $\beta$ AP may impair context conditioning, while learning to sensory cues is not impaired but develops more slowly.

\section{DISCUSSION}

A large body of evidence implicates $\beta A P$ and other derivatives of the evolutionarily highly conserved APP in the pathogenesis 
A

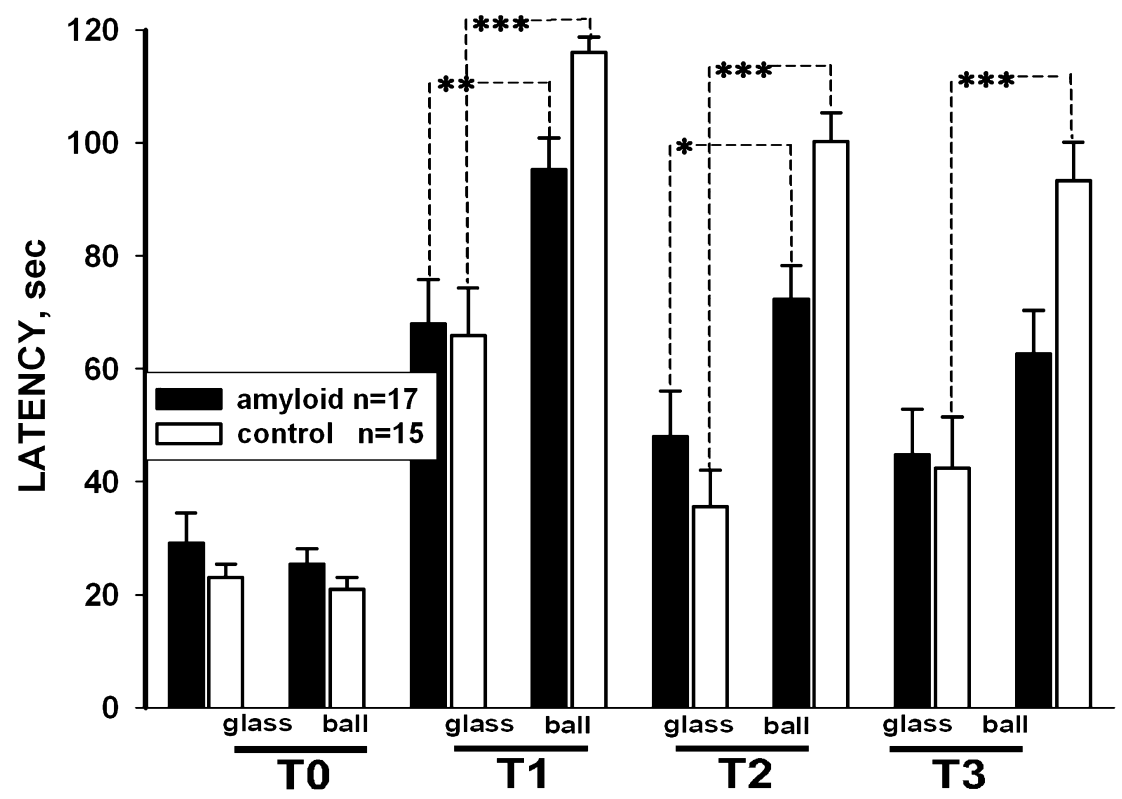

B

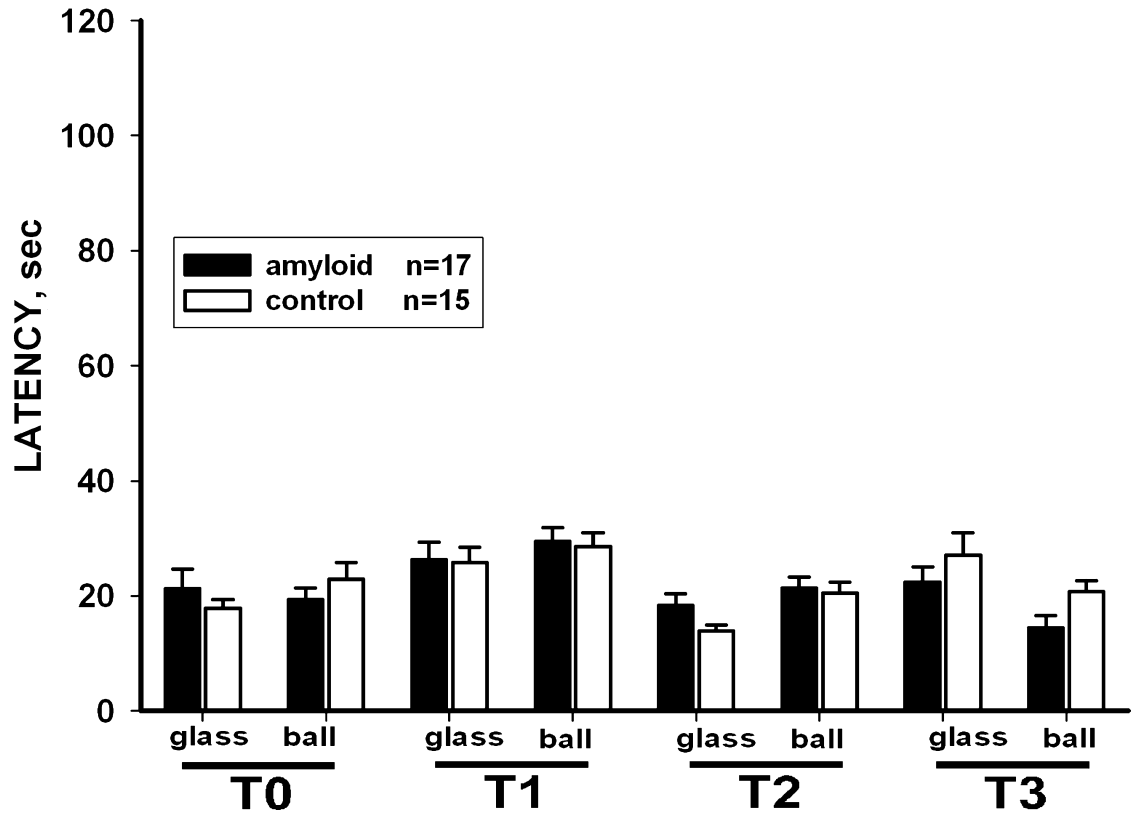

Figure 4 | Changes in latency of conditioned food grasping in tests performed before learning (T0), after 2 (T2), 5 (T2) and 14 days (T3) after learning in two contexts: on the ball (reinforced context) and on the glass. (A) The averaged latency of carrot bites in control snails $(n=15)$ and $\beta A P$-injected snails $(n=17) . Y$-axis - latency period (seconds) of first bite of the carrot. (B) The latency of cabbage bites in control snails $(n=15)$ and $\beta$ AP-injected snails $(n=17)$. $Y$-axis - latency period (seconds) of cabbage bites. ${ }^{*} p<0.05, * * p<0.01, * * * p<0.001$.

of AD. However, the functional relationship of APP and its proteolytic derivatives to behavioral plasticity is not well known. Diverse models have been established to test the amyloid hypothesis in AD. Among these, initial attempts have been made to use invertebrates to this end. The nematode Caenorhabditis elegans was utilized as an in vivo model to correlate the $\beta \mathrm{AP}$ expression with its toxicity to provide a better understanding of the cellular processes underlying AD (Gutierrez-Zepeda and Luo, 2004). So far snails have not been used to study $\beta$ AP-induced neurotoxicity. A single preliminary report demonstrated that $\beta A P$ inhibited GABA-induced $\mathrm{Cl}^{-}$-currents in identified neurons ( $\mathrm{R} 9$ and R12) of Aplysia kurodai without affecting the resting membrane conductance or holding current (Sawada and Ichinose, 1996).
It was shown previously that some changes in synaptic plasticity and behavioral performance can be elicited in terrestrial snails nervous system by application of $\beta A P$ (Korshunova et al., 2007; Samarova et al., 2005). Observed changes in neural and behavioral parameters under $\beta A P$ in these studies served as a basis for the present experiments.

Results described in the present report clearly demonstrate that AP interferes with the learning process but does not fully abolish food-aversion learning. In a separate series of experiments, we tested whether the injection of $\beta$ AP interferes with an important component of associative learning - context memory. We found that $\beta$ AP-injected snails shocked in one context significantly increased responsiveness in all tested contexts 2 days later, while 
A

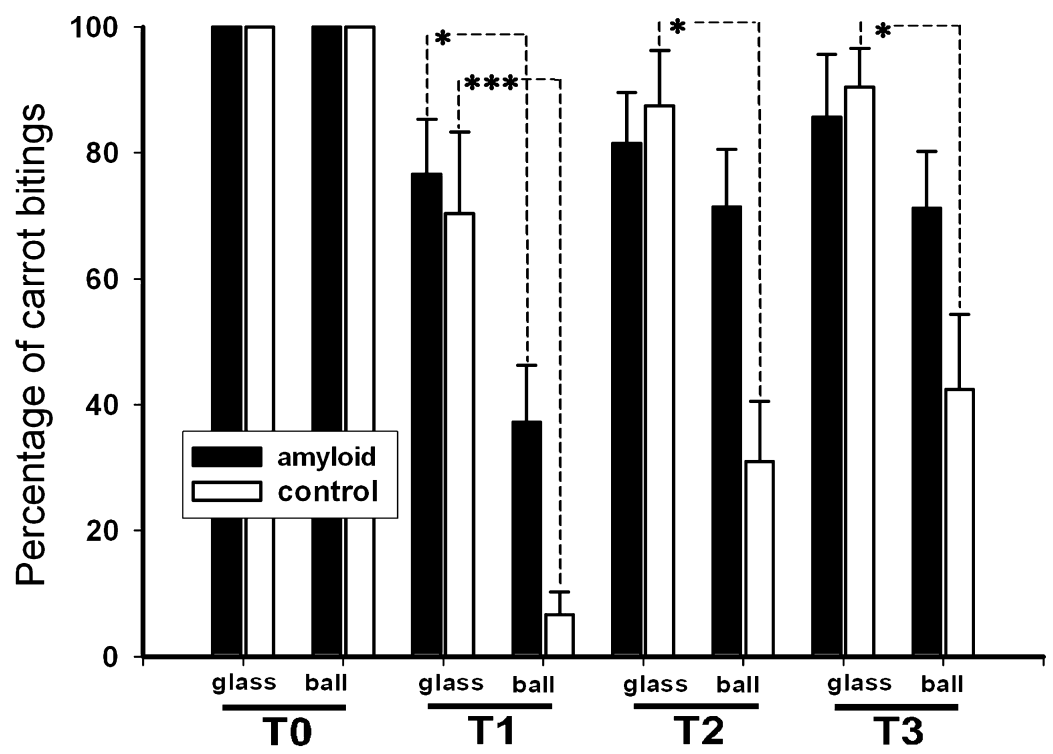

B

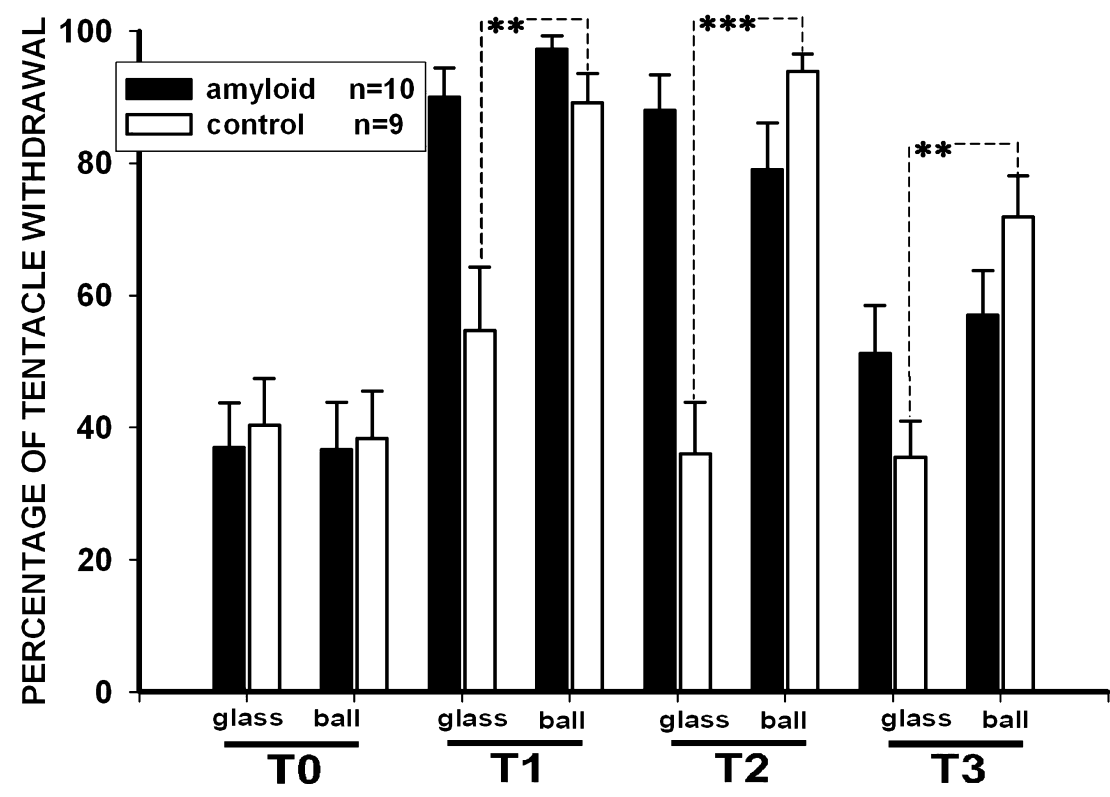

Figure 5 | Changes in quantity of food grasping events (percentage of food presentations number) due to learning, context memory test. (A) Number of carrot bites by control snails $(n=15)$ and $\beta A P$-injected snails $(n=17)$ in two contexts: on the ball (reinforced context) and on the glass. Testing was performed before learning (T0), after 2 (T2), 5 (T2) and 14 days (T3) of rest after learning. $Y$-axis - number of carrot grasps (percentage of presentations), averaged for each snail. (B) Testing of context memory in associatively trained snails; tentacle withdrawal in control snails $(n=9)$ and $\beta$ AP-injected snails $(n=10)$ was estimated in two contexts: on the ball (reinforced context) and on the glass. Testing was performed before learning (T0), after 2 (T2), 5 (T2) and 14 days (T3) after learning. $Y$-axis - amplitude of tentacle withdrawal, percentage of maximal length. $* p<0.05, * * p<0.01, * * * p<0.001$.

sham-injected animals selectively increased responsiveness only in the context in which they were reinforced with electric shocks by responding similarly both in the aversively reinforced and in the neutral context, while when tested with the CS.

The main unanswered question in our results is why in response to tactile stimulus (not involved in conditioning) the $\beta A P-i n j e c t e d$ animals demonstrated a complete lack of context memory by responding similarly both in the aversively reinforced and in the neutral context, while when tested with the CS (carrot) on the 3rd day after the associative training, the same snails demonstrated an ability to distinguish two context situations (Figures 4A and 5A). This ability is worse than in sham-injected animals, but it is statistically significant. During training, the snails learn to associate shocks (reinforcement) with a specific context, and food odor with reinforcement and context. It may be suggested that the association of reinforcement, context and food odor (conditioned stimulus) is different from the association of reinforcement and context due to differences in cellular mechanisms of context and cued memories.

Our results suggest that the neurotoxic fragment of $\beta A P$ (25-35) may play a significant role in behavioral plasticity and memory by chronically impairing only one component of memory - the context memory.

\section{CELLULAR MECHANISMS OF AP EFFECTS}

In Helix, it has been previously shown that after the 5,7-DHT treatment (ablating the serotonergic neurons) both the sensitization of the withdrawal reaction and development of the 
associative aversive conditioning were impaired (Balaban et al., 1987). The feeding behavior of 5,7-DHT-injected intact animals was apparently normal, as were also the electrophysiological responses of the investigated neurons to single feeding and noxious stimuli. This indicates that the absence of this type of associative learning is not due to changes of responsiveness of the neurons taking part in the feeding and aversive behavior, respectively.

The 5,7-DHT treatment after elaboration of aversive conditioning in Helix does not impair the conditioned responses to cued stimuli (Balaban et al., 1987). This result suggests that the 5-HT-containing cells participate in associative learning during the consolidation phase of the conditioned food-aversion reflex, but are not necessary during its retrieval.

We tested a possibility of involvement of the 5-HT-containing neurons, which modulate the network underlying avoidance responses (Zakharov et al., 1995), in contextual conditioning. Injection of 5,7-DHT resulted in a complete loss of context memory. Only responses of vehicle-injected sensitized snails differed significantly in both environments (Balaban, 2002). This result suggests that the 5-HT-ergic neurons are necessary for development, retrieval and/or maintenance of contextual conditioning.

Observed in the present study changes in behavioral performance under $\beta$ AP were similar to behavioral changes under selective blockade of serotonergic system in the snail (blockade of context memory, Balaban et al., 1987). It suggests possible involvement of serotonergic system in behavioral changes observed under influence of $\beta$ AP. In conformity with this, earlier it was shown that the $\beta \mathrm{AP}$ application in vitro reduces sensitization of synaptic responses (which is considered to be a serotonin-dependent mechanism for behavioral sensitization), and behavioral sensitization under $\beta$ AP (Korshunova et al., 2007; Samarova et al., 2005). In the present study, we have monitored the $\beta A P$-induced changes in behavioral performance in terrestrial snails. For the first time we demonstrate that $\beta A P$ significantly and selectively impairs a component of learning - context conditioning, while learning to cued stimuli is different from normal but is not impaired. The $\beta A P$-injected animals exhibited faster decay of retention than those in the control group (Figure 3A), which may be explained by importance of context memory for acquisition and retention.

\section{CONFLICT OF INTEREST STATEMENT}

The authors declare that the research was conducted in the absence of any commercial or financial relationships that could be construed as a potential conflict of interest.

\section{ACKNOWLEDGEMENTS}

Supported by Russian Foundation for Basic Research grant 07-04-01292a, Program of Biology Division of Russian Academy of Sciences.

\section{REFERENCES}

Balaban, P. M. (1993). Behavioral neurobiology of learning in terrestrial snails. Prog. Neurobiol. 41, 1-19.

Balaban, P. M. (2002). Cellular mechanisms of behavioral plasticity in terrestrial snail. Neurosci. Biobehav. Rev. 26, 597-630.

Balaban, P., and Bravarenko, N. (1993). Long-term sensitization and environmental conditioning in terrestrial snails. Exp. Brain Res. 96, 482-493.

Balaban, P. M., Vehovszky, A., Maximova, O. A., and Zakharov, I. S. (1987). Effect of 5,7-DHT on the food-aversive conditioning in the snail Helix lucorum L. Brain Res. 404, 201-210.

Bartoo, G. T., Nochlin, D., Chang, D., Kim, Y., and Sumi, S. M. (1997). The mean A beta load in the hippocampus correlates with duration and severity of dementia in subgroups of Alzheimer disease. J. Neuropathol. Exp. Neurol. 56, 531-540.

Gutierrez-Zepeda, A., and Luo, Y. (2004). Testing the amyloid toxicity hypothesis of Alzheimer's disease in transgenic Caenorhabditis elegans model. Front. Biosci. 9, 3333-3338.

Johnstone, E. M., Chaney, M. O., Norris, F. H., Pascual, R., and Little, S. P. (1991). Conservation of the sequence of the Alzheimer's disease amyloid peptide in dog, polar bear and five other mammals by cross-species polymerase chain reaction analysis. Brain Res. Mol. Brain Res. 10, 299-305.

Kamenetz, F., Tomita, T., Hsieh, H., Seabrook, G., Borchelt, D., Iwatsubo, T., Sisodia, S., and Malinow, R. (2003). APP processing and synaptic function. Neuron 37, 925-937.

Kerkut, G. A. (1989). Studying the isolated central nervous system; a report on 35 years: more inquisitive than acquisitive. Comp. Biochem. Physiol. A 93, 9-24.

Korshunova, T. A., Samarova, E. I., Bravarenko, N. I., and Balaban, P. N. (2007). Beta-amyloid peptide influences behavioral plasticity in terrestrial snail. $Z h$. Vyssh. Nerv. Deiat. Im. I P Pavlova 57, 229-236.

Naslund, J., Haroutunian, V., Mohs, R., Davis, K. L., Davies, P., Greengard, P., and Buxbaum, J. D. (2000). Correlation between elevated levels of amyloid betapeptide in the brain and cognitive decline. JAMA 283, 1571-1577.

Samarova, E. I., Bravarenko, N. I., Korshunova, T. A., Gulyaeva, N. V., Palotas, A., and Balaban, P. M. (2005). Effect of beta-amyloid peptide on behavior and synaptic plasticity in terrestrial snail. Brain Res. Bull. 67, 40-45.

Sawada, M., and Ichinose, M. (1996). Amyloid- $\beta$ proteins reduce the GABAinduced $\mathrm{Cl}^{-}$current in identified Aplysia neurons. Neurosci. Lett. 213, 213-215.

Schulz, P. E. (1996). Beta-peptides enhance the magnitude and probability of long-term potentiation. Abstr. Soc. Neurosci. 22, 2111.

Selkoe, D. J. (2000). Toward a comprehensive theory for Alzheimer's disease. Hypothesis: Alzheimer's disease is caused by the cerebral accumulation and cytotoxicity of amyloid $\beta$-protein. Ann. NY Acad. Sci. 924, 17-25.

Seubert, P., Vigo-Pelfrey, C., Esch, F., Lee, M., Dovey, H., Davis, D., Sinha, S., Schlossmacher, M., Whaley, J., Swindlehurst, C., McCormack, R., Wolfert, R., Selkoe, D., Lieberberg, I., and Schenk, D. (1992). Isolation and quantification of soluble Alzheimer's $\beta$-peptide from biological fluids. Nature 359, 325-327.

Stepanichev, M. Iu., Moiseeva, Iu. V., Lazareva, N. A., and Guliaeva, N. V. (2004). Effects of amyloid beta peptide (25-35) on the behavior of rats in a radial maze. Zh. Vyssh. Nerv. Deiat. Im. I P Pavlova 54, 382-389.

Turner, P. R., O'Connor, K., Tate, W. P., and Abraham, W. C. (2003). Roles of amyloid precursor protein and its fragments in regulating neural activity, plasticity and memory. Prog. Neurobiol. 70, 1-32.

Wasco, W., Gurubhagavatula, S., and Tanzi, R. E. (1995). Molecular characterization of the members of the APP-like gene family. In Research Advances in Alzheimers's Disease and Related Disorders, K. Iqbal, J. A. Mortimer, B. Winblad and H. M. Wisniewski, eds (Chichester, Wiley), pp. 657-667.

Wu, J., Anwyl, R., and Rowan, M. J. (1995). $\beta$-Amyloid selectively augments NMDA receptor-mediated synaptic transmission in rat hippocampus. Neuroreport 6, 2409-2413.

Zakharov, I. S., Ierusalimsky, V. N., and Balaban, P. M. (1995). Pedal serotonergic neurons modulate the synaptic input of withdrawal interneurons in Helix. Invertebr. Neurosci. 1, 41-52. 